\title{
Fixed point results in the generation of Julia and Mandelbrot sets
}

\author{
Waqas Nazeer', Shin Min Kang ${ }^{2 *}$, Muhmmad Tanveer ${ }^{3}$ and Abdul Aziz Shahid ${ }^{3}$
}

\author{
"Correspondence: \\ smkang@gnu.ac.kr \\ ${ }^{2}$ Department of Mathematics and \\ RINS, Gyeongsang National \\ University, Jinju, 660-701, Korea \\ Full list of author information is \\ available at the end of the article
}

\begin{abstract}
The aim of this paper is to establish some fixed point results in the generation of Julia and Mandelbrot sets by using Jungck Mann and Jungck Ishikawa iterations with s-convexity.
\end{abstract}

Keywords: Julia set; Mandelbrot set; Jungck Mann iteration; Jungck Ishikawa iteration

\section{Introduction}

In mathematics, the fractal geometry has presented some attractive complex graphs and objects to computer graphics. Fractal is a Latin word, derived from the word 'fractus' which means 'broken'. First time the term fractal was used by a young mathematician Gaston Julia [1], when he was studying Cayley's problem related to the behavior of Newton's method in complex plane. Julia introduced the concept of iterative function system (IFS), and, by using it, Julia derived the Julia set in 1919. After that, in 1975, Benoit Mandelbrot extended the ideas of Julia. He introduced the Mandelbrot set by using the complex function $z^{2}+$ $c$ with using $z$ as a complex function and $c$ as a complex parameter [2-6]. The fractal structure of Mandelbrot and Julia sets has been demonstrated for quadratic, cubic and higher degree polynomials, by using the Picard orbit which is an application of one-step feedback process [7].

Julia and Mandelbrot sets have been studied under the effect of noises [1-17] arising in the objects. In 2004, Rani and Kumar [5, 16] introduced superior iterates (a two-step feedback process) in the study of fractal theory and created superior Julia and Mandelbrot sets. Later on, in a series of papers Rani et al. generated and analyzed superior Julia and superior Mandelbrot sets for quadratic [3-6, 13-17], cubic [10], and $n$th degree [3, 5, 8, 13-16] complex polynomials. After creation of superior Mandelbrot sets, Negi and Rani [4] collected the properties of midgets of quadratic superior Mandelbrot sets. Negi and Rani [3] simulated the behavior of Julia sets using switching processes. Superior Julia and superior Mandelbrot sets have also been studied under the effect of noises [2-6, 8, 9, 1317]. Chauhan et al. [8, 9] obtained new Julia and Mandelbrot sets via Ishikawa iterates (an example of three-step feedback process). Kang et al. [18] introduced Julia and Mandelbrot sets in Jungck Mann and Jungck Ishikawa orbits.

Mandelbrot set serves as a lexicon for the Julia set. The location of the parameter c within the Mandelbrot set furnishes information on properties of the corresponding Julia 
set. There are similarities between magnified positions of the Mandelbrot set, and the corresponding filled Julia set holds only near certain $c$ values such as the central junctions of the antenna. These are $c$ values, for which 0 is eventually periodic; such $c$ values are called Misiurewicz points [7].

It is a well-known fact that $s$-convexity and Ishikawa iteration play a vital role in the development of geometrical pictures of fractal sets [19]. Further, we know very well about the applications of fractal sets in cryptography and other useful areas in our modern era. In this paper, we deal with generalization of $s$-convexity, approximate convexity and the results of Bernstein and Doetsch [20]. The concept of $s$-convexity and rational $s$-convexity was introduced by Breckner and Orbán [21]. In 1978 Breckner and Orbán [21] and Hudzik and Maligranda [22] proved that $s$-convex functions are nonnegative when $0<s<1$; moreover, the set of $s$-convex functions increases as s decreases.

In 1994, Hudzik and Maligranda [22] discussed a few results related with $s$-convex functions in the second sense, and some new results about Hadamard's inequality for $s$-convex functions were discussed by Alomari and Darus [23, 24] and Kirmaci et al. [25]. In 1999, Dragomir and Fitzpatrick [26] proved a variant of Hermite-Hadamard's inequality for $s$ convex functions in the second sense. Takahashi [27] first introduced a notion of convex metric space, which is a more general space, and each linear normed space is a special example of the space. Very recently Rana et al. [19] discussed the dynamics of Ishikawa iteration procedure. Recently Ojha and Mishra [28] discussed an application of a fixed point theorem for $s$-convex function.

In this paper, we establish some new fixed point results in the generation of Julia and Mandelbrot sets by using Jungck Mann and Jungck Ishikawa iterations with $s$ convexity. We define the Jungck Mann and Jungck Ishikawa orbits and escape criterions for quadratic, cubic and nth degree complex polynomials by using Jungck Mann and Jungck Ishikawa iterations with $s$-convexity.

\section{Preliminaries}

Definition 2.1 (Mandelbrot set [2-6]) The Mandelbrot set $M$ for the quadratic $Q_{c}(z)=$ $z^{2}+c$ is defined as the collection of all $c \in C$ for which the orbit of the point 0 is bounded, that is,

$$
M=\left\{c \in C:\left\{Q_{c}^{n}(0)\right\} ; n=0,1,2, \ldots\right\}
$$

is bounded. An equivalent formulation is

$$
M=\left\{c \in C:\left\{Q_{c}^{n}(0) \text { does not tend to } \infty \text { as } n \rightarrow \infty\right\}\right\} .
$$

We choose the initial point 0 , as 0 is the only critical point of $Q_{c}$.

Definition 2.2 (Julia set [1]) The attractor basin of infinity is never all of $C$ since $f_{c}$ has fixed points $z_{f}=1 / 2 \pm \sqrt{1 / 4+c}$ (and also points of period $n$ that satisfy a polynomial equation of degree $2^{n}$, namely $\left.f^{n}(z)=z\right)$. The nonempty, compact boundary of the attractor basin of infinity is called the Julia set of $f_{c}$,

$$
J_{c}=\partial A_{\infty}(c) .
$$


Definition 2.3 (Filled Julia set $[1,7,10-17])$ The filled in Julia set of the function $f$ is defined as

$$
K(f)=\left\{z \in C: f^{k}(z) \rightarrow \infty\right\} .
$$

Definition 2.4 ([1, 7, 10-17]) The Julia set of the function $f$ is defined to be the boundary of $K(f)$, i.e.,

$$
J(f)=\partial K(f)
$$

Definition 2.5 [7] Let $\left\{z_{n}: n=1,2,3,4, \ldots\right\}$ denoted by $\left\{z_{n}\right\}$ be a sequence of complex numbers. Then we say $\operatorname{Lim}_{n \rightarrow \infty} z_{n}=\infty$ if for given $M>0$, there exists $N>0$ such that for all $n>N$, we must have $\left|z_{n}\right|>M$. Thus all the values of $z_{n}$ lie outside a circle of radius $M$ for sufficiently large values of $n$. Let

$$
Q(z)=a_{0} z^{n}+a_{1} z^{n-1}+a_{2} z^{n-2}+\cdots+a_{n-1} z^{1}+a_{n} z^{0} ; \quad a_{0} \neq 0
$$

be a polynomial of degree $n$, where $n \geq 2$. The coefficients are allowed to be complex numbers. In other words, it follows that $Q_{c}(z)=z^{2}+c$.

Definition 2.6 (Picard orbit [7]) Let $X$ be a nonempty set and $f: X \rightarrow X$. For any point $x_{0} \in X$, the Picard orbit is defined as the set of iterates of a point $x_{0}$, that is,

$$
O\left(f, x_{0}\right)=\left\{x_{n} ; x_{n}=f\left(x_{n-1}\right), n=1,2,3, \ldots\right\} .
$$

Definition 2.7 (Jungck Mann orbit [29]) Let us consider the sequence $\left\{x_{n}\right\}$ of iterates for any initial point $x_{0} \in X$ such that

$$
\left\{S x_{n+1:} S x_{n+1}=(1-\alpha)^{s} S x_{n}+\alpha^{s} T x_{n}\right\}
$$

where $\alpha, s \in(0,1)$ for $n=0,1,2, \ldots$ The above sequence of iterates with $s$-convexity is called Jungck Mann orbit, denoted by $J M O$, which is a function of four tuple $\left(T, x_{0}, \alpha, s\right)$.

Definition 2.8 (Jungck Ishikawa orbit [29]) Let us consider the sequence $\left\{x_{n}\right\}$ of iterates for any initial point $x_{0} \in X$ such that

$$
\begin{aligned}
\left\{S x_{n+1}: S x_{n+1}\right. & =(1-\alpha)^{s} S x_{n}+\alpha^{s} T y_{n} ; \\
S y_{n} & \left.=(1-\beta)^{s} S x_{n}+\beta^{s} T x_{n}\right\},
\end{aligned}
$$

where $\alpha, \beta, s \in(0,1)$ for $n=0,1,2, \ldots$. The above sequence of iterates with $s$-convexity is called Jungck Ishikawa orbit, denoted by $J I O$, which is a function of six tuple $\left(T, x_{0}, \alpha, \beta, s\right)$.

Remark 2.9 The JIO reduces to:

1. The Jungck Mann orbit when $\beta=0$;

2. The Jungck orbit when $\beta=0$ and $\alpha=1$. 
In nonlinear dynamics, we have two different types of points. Points that leave the interval after a finite number are in a stable set of infinity. Points that never leave the interval after any number of iterations have bounded orbits. So, an orbit is bounded if there exists a positive real number such that the modulus of every point in the orbit is less than this number. The collection of points that are bounded, i.e., there exists $M$ such that $\left|Q^{n}(z)\right| \leq M$, for all $n$, is called a prisoner set, while the collection of points that are in the stable set of infinity is called an escape set. Hence, the boundary of the prisoner set is simultaneously the boundary of the escape set and that is the Mandelbrot set for $Q$.

\section{Escape criterions for the complex polynomials in Jungck Mann orbit}

The escape criterion is the key to generate the Julia sets and Mandelbrot sets. In this paper, we prove the escape criterions of Julia and Mandelbrot sets for quadratics, cubics and the higher degree complex polynomials in Jungck Mann orbit.

\subsection{Escape criterion for the quadratic complex polynomials}

For quadratic complex polynomial $p(z)=z^{2}-a z+c$, we will choose $T z=z^{2}+c$ and $S z=a z$, where $a$ and $c$ are complex numbers.

Theorem 3.1 Assume that $|z| \geq|c|>\frac{2(1+|a|)}{s \alpha}$, where $0<\alpha, s<1$ and $c$ is a complex parameter. Define

$$
\begin{aligned}
& S z_{1}=(1-\alpha)^{s} S z+\alpha^{s} T z, \\
& \vdots \\
& S z_{n}=(1-\alpha)^{s} S z_{n-1}+\alpha^{s} T z_{n-1},
\end{aligned}
$$

where $S z$ is injective, $T z$ is a quadratic polynomial and $n=2,3,4, \ldots$, then $\left|z_{n}\right| \rightarrow \infty$ as $n \rightarrow \infty$.

Proof Let $T z=z^{2}+c$ and $z_{0}=z$, then we have

$$
S z_{n}=(1-\alpha)^{S} S z_{n-1}+\alpha^{s} T z_{n-1}
$$

implies

$$
\begin{aligned}
\left|S z_{1}\right| & =\left|(1-\alpha)^{s} S z+\alpha^{s} T z\right| \\
& =\left|(1-\alpha)^{s} a z+\alpha^{s}\left(z^{2}+c\right)\right| \\
& =\left|(1-\alpha)^{s} a z+(1-(1-\alpha))^{s}\left(z^{2}+c\right)\right| .
\end{aligned}
$$

Using binomial series up to linear terms of $\alpha$ and $(1-\alpha)$, we get

$$
\begin{aligned}
\left|S z_{1}\right| & =\left|(1-s \alpha) a z+(1-s(1-\alpha))\left(z^{2}+c\right)\right| \\
& \geq(1-s(1-\alpha))\left|z^{2}+c\right|-|(1-s \alpha) a z| \\
& \geq(s-s(1-\alpha))\left|z^{2}+c\right|-|(1-s \alpha) a z|, \quad s<1
\end{aligned}
$$




$$
\begin{aligned}
& \geq s \alpha\left|z^{2}\right|-s \alpha|c|-|a z|+|s \alpha a z| \\
& \geq s \alpha\left|z^{2}\right|-s \alpha|z|-|a z|+|s \alpha a z|, \quad|z| \geq|c| \\
& \geq s \alpha\left|z^{2}\right|-s \alpha|z|-|a z|, \quad|a| \geq 0
\end{aligned}
$$

gives us

$$
\begin{aligned}
\left|a z_{1}\right| & \geq s \alpha\left|z^{2}\right|-|z|-|a z|, \quad s \alpha<1 \\
& \geq s \alpha\left|z^{2}\right|-|a||z|-|z| \\
& =s \alpha\left|z^{2}\right|-(1+|a|)|z| \\
& =|z|(s \alpha|z|-(1+|a|)) .
\end{aligned}
$$

Thus

$$
\left|z_{1}\right| \geq|z|\left(\frac{s \alpha|z|}{1+|a|}-1\right)
$$

since $|z| \geq|c|>\frac{2(1+|a|)}{s \alpha}$, therefore there exists $\lambda>0$ such that $\frac{s \alpha|z|}{1+|a|}-1>1+\lambda$. Consequently, $\left|z_{1}\right|>(1+\lambda)|z|$. In particular, $\left|z_{n}\right|>|z|$. So we may apply the same argument repeatedly to find $\left|z_{n}\right|>(1+\lambda)^{n}|z|$. Thus, the orbit of $z$ tends to infinity. This completes the proof.

Corollary 3.2 Suppose that $|c|>\frac{2(1+|a|)}{s \alpha}$, then the orbit of Jungck Mann $\operatorname{JMO}\left(T_{c}, 0, \alpha, s\right)$ escapes to infinity.

In the proof of the theorem we used the fact that $|z| \geq|c|>\frac{2(1+|a|)}{s \alpha}$. Hence the following corollary is the refinement of the escape criterion discussed in the above theorem.

Corollary 3.3 (Escape criterion) Let $|z|>\max \left\{|c|, \frac{2(1+|a|)}{s \alpha}\right\}$, then $\left|z_{n}\right|>(1+\lambda)^{n}|z|$ and $\left|z_{n}\right| \longrightarrow \infty$ as $n \rightarrow \infty$.

Corollary 3.4 Suppose that $\left|z_{k}\right|>\max \left\{|c|, \frac{2(1+|a|)}{s \alpha}\right\}$ for some $k \geq 0$. Then $\left|z_{k+1}\right|>(1+\lambda)^{n}\left|z_{k}\right|$ and $\left|z_{k+1}\right| \longrightarrow \infty$ as $n \rightarrow \infty$.

This corollary gives us an algorithm for the generation of Julia sets and Mandelbrot sets of $T_{c}$. Given any point $|z| \leq|c|$, we have computed the orbit 'JMO' of $z$. If for some $n,\left|z_{n}\right|$ lies outside the circle of radius $\max \left\{|c|, \frac{2(1+|a|)}{s \alpha}\right\}$, we guarantee that the orbit escapes. Hence, $z$ is not in the Julia sets and also it is not in the Mandelbrot sets. On the other hand, if $\left|z_{n}\right|$ never exceeds this bound, then by definition of the Julia sets and the Mandelbrot sets we can make extensive use of this algorithm in the next section.

\subsection{Escape criterion for the cubic complex polynomials}

For cubic complex polynomial $p(z)=z^{3}-a z+c$, we will choose $T z=z^{3}+c$ and $S z=a z$, where $a$ and $c$ are complex numbers.

Theorem 3.5 Assume that $|z| \geq|c|>\left(\frac{2(1+|a|)}{s \alpha}\right)^{\frac{1}{2}}$, where $0<\alpha, s<1$ and $c$ is a complex parameter. Define

$$
S z_{1}=(1-\alpha)^{s} S z+\alpha^{s} T z,
$$




$$
\begin{aligned}
& \vdots \\
& S z_{n}=(1-\alpha)^{s} S z_{n-1}+\alpha^{s} T z_{n-1},
\end{aligned}
$$

where $S z$ is injective, Tz is a cubic polynomial and $n=2,3,4, \ldots$, then $\left|z_{n}\right| \rightarrow \infty$ as $n \rightarrow \infty$.

Proof Let $T z=z^{3}+c$ and $z_{0}=z$, then we have

$$
S z_{n}=(1-\alpha)^{s} S z_{n-1}+\alpha^{s} T z_{n-1}
$$

implies

$$
\begin{aligned}
\left|S z_{1}\right| & =\left|(1-\alpha)^{s} S z+\alpha^{s} T z\right| \\
& =\left|(1-\alpha)^{s} a z+\alpha^{s}\left(z^{3}+c\right)\right| \\
& =\left|(1-\alpha)^{s} a z+(1-(1-\alpha))^{s}\left(z^{3}+c\right)\right| .
\end{aligned}
$$

Using binomial series up to linear terms of $\alpha$ and $(1-\alpha)$, we get

$$
\begin{aligned}
\left|S z_{1}\right| & =\left|(1-s \alpha) a z+(1-s(1-\alpha))\left(z^{3}+c\right)\right| \\
& \geq(1-s(1-\alpha))\left|z^{3}+c\right|-|(1-s \alpha) a z| \\
& \geq(s-s(1-\alpha))\left|z^{3}+c\right|-|(1-s \alpha) a z|, \quad s<1 \\
& \geq s \alpha\left|z^{3}\right|-s \alpha|c|-|a z|+|s \alpha a z| \\
& \geq s \alpha\left|z^{3}\right|-s \alpha|z|-|a z|+|s \alpha a z|, \quad|z| \geq|c| \\
& \geq s \alpha\left|z^{3}\right|-s \alpha|z|-|a z|, \quad|a| \geq 0
\end{aligned}
$$

gives us

$$
\begin{aligned}
\left|a z_{1}\right| & \geq s \alpha\left|z^{3}\right|-|z|-|a z|, \quad s \alpha<1 \\
& \geq s \alpha\left|z^{3}\right|-|a||z|-|z| \\
& =s \alpha\left|z^{3}\right|-(1+|a|)|z| \\
& =|z|\left(s \alpha\left|z^{2}\right|-(1+|a|)\right) .
\end{aligned}
$$

Thus

$$
\left|z_{1}\right| \geq|z|\left(\frac{s \alpha\left|z^{2}\right|}{1+|a|}-1\right)
$$

since $|z| \geq|c|>\left(\frac{2(1+|a|)}{s \alpha}\right)^{\frac{1}{2}}$, therefore there exists $\lambda>0$ such that $\frac{s \alpha\left|z^{2}\right|}{1+|a|}-1>1+\lambda$. Consequently, $\left|z_{1}\right|>(1+\lambda)|z|$. In particular, $\left|z_{n}\right|>|z|$. So we may apply the same argument repeatedly to find $\left|z_{n}\right|>(1+\lambda)^{n}|z|$. Thus, the orbit of $z$ tends to infinity. This completes the proof.

Corollary 3.6 Suppose that $|c|>\left(\frac{2(1+|a|)}{s \alpha}\right)^{\frac{1}{2}}$, then the orbit of Jungck Mann $\operatorname{JMO}\left(T_{c}, 0, \alpha, s\right)$ escapes to infinity. 
In the proof of the theorem we used the fact that $|z| \geq|c|>\left(\frac{2(1+|a|)}{s \alpha}\right)^{\frac{1}{2}}$. Hence the following corollary is the refinement of the escape criterion discussed in the above theorem.

Corollary 3.7 (Escape criterion) Let $|z|>\max \left\{|c|,\left(\frac{2(1+|a|)}{s \alpha}\right)\right\}$, then $\left|z_{n}\right|>(1+\lambda)^{n}|z|$ and $\left|z_{n}\right| \rightarrow \infty$ as $n \rightarrow \infty$.

Corollary 3.8 Suppose that $\left|z_{k}\right|>\max \left\{|c|,\left(\frac{2(1+|a|)}{s \alpha}\right)^{\frac{1}{2}}\right\}$ for some $k \geq 0$. Then $\left|z_{k+1}\right|>(1+$ $\lambda)^{n}\left|z_{k}\right|$ and $\left|z_{k+1}\right| \longrightarrow \infty$ as $n \rightarrow \infty$.

This corollary gives us an algorithm for the generation of Julia sets and Mandelbrot sets of $T_{c} z=z^{3}+c$.

\subsection{Escape criterion for higher degree complex polynomials}

For higher degree complex polynomial $p(z)=z^{n}-a z+c$, we will choose $T z=z^{n}+c$ and $S z=a z$, where $n=2,3,4, \ldots, a$ and $c$ are complex numbers.

Theorem 3.9 Assume that $|z| \geq|c|>\left(\frac{2(1+|a|)}{s \alpha}\right)^{\frac{1}{n-1}}$, where $0<\alpha, s<1$ and $c$ is a complex parameter. Define

$$
\begin{aligned}
& S z_{1}=(1-\alpha)^{s} S z+\alpha^{s} T z, \\
& \vdots \\
& S z_{n}=(1-\alpha)^{s} S z_{n-1}+\alpha^{s} T z_{n-1},
\end{aligned}
$$

where $S z$ is injective, $T z=z^{n}+c$ and $n=2,3,4, \ldots$, then $\left|z_{n}\right| \rightarrow \infty$ as $n \rightarrow \infty$.

Proof To prove the theorem, we follow the mathematical induction. For $n=2, T z=z^{2}+c$, so the escape criterion is $|z|>\max \left\{|c|, \frac{2(1+|a|)}{s \alpha}\right\}$. For $n=3, T z=z^{3}+c$, so the escape criterion is $|z|>\max \left\{|c|,\left(\frac{2(1+|a|)}{s \alpha}\right)^{1 / 2}\right\}$. Hence the theorem is true for $n=2,3, \ldots$. Now suppose that the theorem is true for any $n$. Let $T z=z^{n+1}+c, z_{0}=z$ and $|z| \geq|c|>\left(\frac{2(1+|a|)}{s \alpha}\right)^{\frac{1}{n}}$ exist, then we have

$$
S z_{n}=(1-\alpha)^{s} S z_{n-1}+\alpha^{s} T z_{n-1}
$$

implies

$$
\begin{aligned}
\left|S z_{1}\right| & =\left|(1-\alpha)^{s} S z+\alpha^{s} T z\right| \\
& =\left|(1-\alpha)^{s} a z+\alpha^{s}\left(z^{n+1}+c\right)\right| \\
& =\left|(1-\alpha)^{s} a z+(1-(1-\alpha))^{s}\left(z^{n+1}+c\right)\right| .
\end{aligned}
$$

Using binomial series up to linear terms of $\alpha$ and $(1-\alpha)$, we get

$$
\begin{aligned}
\left|S z_{1}\right| & =\left|(1-s \alpha) a z+(1-s(1-\alpha))\left(z^{n+1}+c\right)\right| \\
& \geq(1-s(1-\alpha))\left|z^{n+1}+c\right|-|(1-s \alpha) a z| \\
& \geq(s-s(1-\alpha))\left|z^{n+1}+c\right|-|(1-s \alpha) a z| \quad \text { because } s<1
\end{aligned}
$$




$$
\begin{aligned}
& \geq s \alpha\left|z^{n+1}\right|-s \alpha|c|-|a z|+|s \alpha a z| \\
& \geq s \alpha\left|z^{n+1}\right|-s \alpha|z|-|a z|+|s \alpha a z| \quad \text { because }|z| \geq|c| \\
& \geq s \alpha\left|z^{n+1}\right|-s \alpha|z|-|a z| \quad \text { because }|a| \geq 0
\end{aligned}
$$

gives us

$$
\begin{aligned}
\left|a z_{1}\right| & \geq s \alpha\left|z^{n+1}\right|-|z|-|a z| \quad \text { because } s \alpha<1 \\
& \geq s \alpha\left|z^{n+1}\right|-|a||z|-|z| \\
& =s \alpha\left|z^{n+1}\right|-(1+|a|)|z| \\
& =|z|\left(s \alpha\left|z^{n}\right|-(1+|a|)\right) .
\end{aligned}
$$

Thus

$$
\left|z_{1}\right| \geq|z|\left(\frac{s \alpha\left|z^{n}\right|}{1+|a|}-1\right)
$$

since $|z| \geq|c|>\left(\frac{2(1+|a|)}{s \alpha}\right)^{\frac{1}{n}}$, so that $|z|>\left(\frac{2(1+|a|)}{s \alpha}\right)^{\frac{1}{n}}$. Therefore there exists $\lambda>0$ such that $\frac{s \alpha\left|z^{n}\right|}{1+|a|}-1>1+\lambda$. Consequently, $\left|z_{1}\right|>(1+\lambda)|z|$. In particular, $\left|z_{n}\right|>|z|$. So we may apply the same argument repeatedly to find $\left|z_{n}\right|>(1+\lambda)^{n}|z|$. Thus, the orbit of $z$ tends to infinity. This completes the proof.

Corollary 3.10 Suppose that $|c|>\left(\frac{2(1+|a|)}{s \alpha}\right)^{\frac{1}{n-1}}$, then the orbit $J M O\left(T_{c}, 0, \alpha, s\right)$ escapes to infinity.

Corollary 3.11 (Escape criterion) Suppose that $\left|z_{k}\right|>\max \left\{|c|,\left(\frac{2(1+|a|)}{s \alpha}\right)^{\frac{1}{n-1}}\right\}$ for some $k \geq 0$. Then $\left|z_{k+1}\right|>(1+\lambda)^{n}\left|z_{k}\right|$ and $\left|z_{k+1}\right| \longrightarrow \infty$ as $n \rightarrow \infty$.

This corollary gives us an algorithm for the generation of Julia sets and Mandelbrot sets of $T_{c} z=z^{n}+c$.

\section{Escape criterions for the complex polynomials in Jungck Ishikawa orbit}

Now we prove the escape criterions of Julia and Mandelbrot sets for quadratics, cubics and the higher degree complex polynomials in Jungck Ishikawa orbit.

\subsection{Escape criterion for the quadratic complex polynomials}

For quadratic complex polynomial $p(z)=z^{2}-a z+c$, we will choose $T z=z^{2}+c$ and $S z=a z$, where $a$ and $c$ are complex numbers.

Theorem 4.1 Assume that $|z| \geq|c|>\frac{2(1+|a|)}{s \alpha},|z| \geq|c|>\frac{2(1+|a|)}{s \beta}$, where $0<\alpha, \beta, s<1$ and $c$ is a complex parameter. Define

$$
\begin{aligned}
& S z_{1}=(1-\alpha)^{s} S z+\alpha^{s} T y, \\
& \vdots \\
& S z_{n}=(1-\alpha)^{s} S z_{n-1}+\alpha^{s} T y_{n-1},
\end{aligned}
$$


where

$$
\begin{aligned}
& S y=(1-\beta)^{s} S z+\beta^{s} T z, \\
& \vdots \\
& S y_{n-1}=(1-\beta)^{s} S z_{n-1}+\beta^{s} T z_{n-1},
\end{aligned}
$$

Sz is injective, Tz is a quadratic polynomial and $n=2,3,4, \ldots$, then $\left|z_{n}\right| \rightarrow \infty$ as $n \rightarrow \infty$.

Proof Let $T z=z^{2}+c$ and for $z_{0}=z$ and $y_{0}=y$, then we have

$$
S y_{n-1}=(1-\beta)^{s} S z_{n-1}+\beta^{s} T z_{n-1},
$$

implies

$$
\begin{aligned}
|S y| & =\left|(1-\beta)^{s} S z+\beta^{s} T z\right| \\
& =\left|(1-\beta)^{s} a z+\beta^{s}\left(z^{2}+c\right)\right| \\
& =\left|(1-\beta)^{s} a z+(1-(1-\beta))^{s}\left(z^{2}+c\right)\right| .
\end{aligned}
$$

Using binomial series up to linear terms of $\alpha$ and $(1-\alpha)$, we get

$$
\begin{aligned}
|S y| & =\left|(1-s \beta) a z+(1-s(1-\beta))\left(z^{2}+c\right)\right| \\
& \geq(1-s(1-\beta))\left|z^{2}+c\right|-|(1-s \beta) a z| \\
& \geq(s-s(1-\beta))\left|z^{2}+c\right|-|(1-s \beta) a z| \quad \text { because } s<1 \\
& \geq s \beta\left|z^{2}\right|-s \beta|c|-|a z|+|s \beta a z| \\
& \geq s \beta\left|z^{2}\right|-s \beta|z|-|a z|+|s \beta a z| \quad \text { because }|z| \geq|c| \\
& \geq s \beta\left|z^{2}\right|-s \beta|z|-|a z| \quad \text { because }|a| \geq 0
\end{aligned}
$$

gives us

$$
\begin{aligned}
|a y| & \geq s \beta\left|z^{2}\right|-|z|-|a z| \quad \text { because } s \beta<1 \\
& \geq s \beta\left|z^{2}\right|-|a||z|-|z| \\
& =s \beta\left|z^{2}\right|-(1+|a|)|z| \\
& =|z|(s \beta|z|-(1+|a|)) .
\end{aligned}
$$

Thus

$$
|y| \geq|z|\left(\frac{s \beta|z|}{1+|a|}-1\right)
$$

Since $|z|>\frac{2(1+|a|)}{s \beta}$ implies $|z|^{2}\left(\frac{s \beta|z|}{1+|a|}-1\right)^{2}>|z|^{2}$. Hence $|y|^{2}>|z|^{2}\left(\frac{s \beta|z|}{1+|a|}-1\right)^{2}>|z|^{2}>s \beta|z|^{2}$. 
For $z_{0}=z$ and $y_{0}=y$, consider

$$
\left|S z_{n}\right|=\left|(1-\alpha)^{s} S z_{n-1}+\alpha^{s} T y_{n-1}\right|
$$

implies

$$
\left|S z_{1}\right|=\left|(1-\alpha)^{s} S z+\alpha^{s} T y\right|
$$

which yields

$$
\begin{aligned}
\left|a z_{1}\right| & =\left|(1-\alpha)^{s} S z+\alpha^{s} T y\right| \\
& =\left|(1-\alpha)^{s} a z+(1-(1-\alpha))^{s}\left(y^{2}+c\right)\right| \\
& \geq(1-s(1-\alpha))\left|y^{2}+c\right|-|(1-s \alpha) a z| \\
& \geq s \alpha\left|y^{2}\right|-(1+|a|)|z| \\
& \geq s^{2} \alpha \beta\left|z^{2}\right|-(1+|a|)|z| \\
& \geq|z|\left(s^{2} \alpha \beta|z|-(1+|a|)\right) .
\end{aligned}
$$

Hence

$$
\left|z_{1}\right| \geq|z|\left(\frac{s^{2} \alpha \beta|z|}{1+|a|}-1\right)
$$

since $|z| \geq|c|>\frac{2(1+|a|)}{s \alpha}$ and $|z| \geq|c|>\frac{2(1+|a|)}{s \beta}$, so that $|z|>\frac{2(1+|a|)}{s^{2} \alpha \beta}$. Therefore there exists $\lambda>0$ such that $\frac{s^{s} \alpha \beta|z|}{1+|a|}-1>1+\lambda$. Consequently, $\left|z_{1}\right|>(1+\lambda)|z|$. In particular, $\left|z_{n}\right|>|z|$. So we may apply the same argument repeatedly to find $\left|z_{n}\right|>(1+\lambda)^{n}|z|$. Thus, the orbit of $z$ tends to infinity. This completes the proof.

Corollary 4.2 Suppose that $|c|>\frac{2(1+|a|)}{s \alpha}$ and $|c|>\frac{2(1+|a|)}{s \beta}$, then the orbit of Jungck Ishikawa $J I O\left(T_{c}, 0, \alpha, \beta, s\right)$ escapes to infinity.

In the proof of the theorem we used the facts that $|z| \geq|c|>\frac{2(1+|a|)}{s \alpha}$ and $|z| \geq|c|>\frac{2(1+|a|)}{s \beta}$. Hence the following corollary is the refinement of the escape criterion discussed in the above theorem.

Corollary 4.3 (Escape criterion) Let $|z|>\max \left\{|c|, \frac{2(1+|a|)}{s \alpha}, \frac{2(1+|a|)}{s \beta}\right\}$, then $\left|z_{n}\right|>(1+\lambda)^{n}|z|$ and $\left|z_{n}\right| \longrightarrow \infty$ as $n \rightarrow \infty$.

Corollary 4.4 Suppose that $\left|z_{k}\right|>\max \left\{|c|, \frac{2(1+|a|)}{s \alpha}, \frac{2(1+|a|)}{s \beta}\right\}$ for some $k \geq 0$. Then $\left|z_{k+1}\right|>(1+$ $\lambda)^{n}\left|z_{k}\right|$ and $\left|z_{k+1}\right| \longrightarrow \infty$ as $n \rightarrow \infty$.

This corollary gives us an algorithm for the generation of Julia sets and Mandelbrot sets of $T_{c}$. Given any point $|z| \leq|c|$, we have computed the orbit 'JIO' of $z$. If for some $n,\left|z_{n}\right|$ lies outside the circle of radius $\max \left\{|c|, \frac{2(1+|a|)}{s \alpha}, \frac{2(1+|a|)}{s \beta}\right\}$, we guarantee that the orbit escapes. Hence, $z$ is not in the Julia sets and also it is not in the Mandelbrot sets. On the other hand, if $\left|z_{n}\right|$ never exceeds this bound, then by definition of the Julia sets and the Mandelbrot sets we can make extensive use of this algorithm in the next section. 


\subsection{Escape criterion for the cubic complex polynomials}

For cubic complex polynomial $p(z)=z^{3}-a z+c$, we will choose $T z=z^{3}+c$ and $S z=a z$, where $a$ and $c$ are complex numbers.

Theorem 4.5 Assume that $|z| \geq|c|>\left(\frac{2(1+|a|)}{s \alpha}\right)^{\frac{1}{2}}$ and $|z| \geq|c|>\left(\frac{2(1+|a|)}{s \beta}\right)^{\frac{1}{2}}$, where $0<\alpha, \beta, s<$ 1 and $c$ is a complex parameter. Define

$$
\begin{aligned}
& S z_{1}=(1-\alpha)^{s} S z+\alpha^{s} T y, \\
& \vdots \\
& S z_{n}=(1-\alpha)^{s} S z_{n-1}+\alpha^{s} T y_{n-1},
\end{aligned}
$$

where

$$
\begin{aligned}
& S y=(1-\beta)^{s} S z+\beta^{s} T z, \\
& \vdots \\
& S y_{n-1}=(1-\beta)^{s} S z_{n-1}+\beta^{s} T z_{n-1},
\end{aligned}
$$

Sz is injective, Tz is a cubic polynomial and $n=2,3,4, \ldots$, then $\left|z_{n}\right| \rightarrow \infty$ as $n \rightarrow \infty$.

Proof Let $T z=z^{3}+c$ and for $z_{0}=z$ and $y_{0}=y$, then we have

$$
S y_{n-1}=(1-\beta)^{s} S z_{n-1}+\beta^{s} T z_{n-1}
$$

implies

$$
\begin{aligned}
|S y| & =\left|(1-\beta)^{s} S z+\beta^{s} T z\right| \\
& =\left|(1-\beta)^{s} a z+\beta^{s}\left(z^{3}+c\right)\right| \\
& =\left|(1-\beta)^{s} a z+(1-(1-\beta))^{s}\left(z^{3}+c\right)\right| .
\end{aligned}
$$

Using binomial series up to linear terms of $\alpha$ and $(1-\alpha)$, we get

$$
\begin{aligned}
|S y| & =\left|(1-s \beta) a z+(1-s(1-\beta))\left(z^{3}+c\right)\right| \\
& \geq(1-s(1-\beta))\left|z^{3}+c\right|-|(1-s \beta) a z| \\
& \geq(s-s(1-\beta))\left|z^{3}+c\right|-|(1-s \beta) a z| \quad \text { because } s<1 \\
& \geq s \beta\left|z^{3}\right|-s \beta|c|-|a z|+|s \beta a z| \\
& \geq s \beta\left|z^{3}\right|-s \beta|z|-|a z|+|s \beta a z| \quad \text { because }|z| \geq|c| \\
& \geq s \beta\left|z^{3}\right|-s \beta|z|-|a z| \quad \text { because }|a| \geq 0
\end{aligned}
$$

gives us

$$
\begin{aligned}
|a y| & \geq s \beta\left|z^{3}\right|-|z|-|a z| \quad \text { because } s \beta<1 \\
& \geq s \beta\left|z^{3}\right|-|a||z|-|z|
\end{aligned}
$$




$$
\begin{aligned}
& =s \beta\left|z^{3}\right|-(1+|a|)|z| \\
& =|z|\left(s \beta\left|z^{2}\right|-(1+|a|)\right) .
\end{aligned}
$$

Thus

$$
|y| \geq|z|\left(\frac{s \beta\left|z^{2}\right|}{1+|a|}-1\right) .
$$

Since $|z| \geq|c|>\left(\frac{2(1+|a|)}{s \beta}\right)^{\frac{1}{2}}$ implies $|z|^{3}\left(\frac{s \beta\left|z^{2}\right|}{1+|a|}-1\right)^{3}>|z|^{3}$. Hence $|y|^{3}>|z|^{3}\left(\frac{s \beta|z|}{1+|a|}-1\right)^{3}>|z|^{3}>$ $s \beta|z|^{3}$.

For $z_{0}=z$ and $y_{0}=y$, consider

$$
\left|S z_{n}\right|=\left|(1-\alpha)^{s} S z_{n-1}+\alpha^{s} T y_{n-1}\right|
$$

implies

$$
\left|S z_{1}\right|=\left|(1-\alpha)^{s} S z+\alpha^{s} T y\right|
$$

which yields

$$
\begin{aligned}
\left|a z_{1}\right| & =\left|(1-\alpha)^{s} S z+\alpha^{s} T y\right| \\
& =\left|(1-\alpha)^{s} a z+(1-(1-\alpha))^{s}\left(y^{3}+c\right)\right| \\
& \geq(1-s(1-\alpha))\left|y^{3}+c\right|-|(1-s \alpha) a z| \\
& \geq s \alpha\left|y^{3}\right|-(1+|a|)|z| \\
& \geq s^{2} \alpha \beta\left|z^{3}\right|-(1+|a|)|z| \\
& \geq|z|\left(s^{2} \alpha \beta\left|z^{2}\right|-(1+|a|)\right) .
\end{aligned}
$$

Hence

$$
\left|z_{1}\right| \geq|z|\left(\frac{s^{2} \alpha \beta\left|z^{2}\right|}{1+|a|}-1\right)
$$

since $|z| \geq|c|>\left(\frac{2(1+|a|)}{s \alpha}\right)^{\frac{1}{2}}$ and $|z| \geq|c|>\left(\frac{2(1+|a|)}{s \beta}\right)^{\frac{1}{2}}$, so that $|z|>\left(\frac{2(1+|a|)}{s^{2} \alpha \beta}\right)^{\frac{1}{2}}$. Therefore there exists $\lambda>0$ such that $\frac{s^{2} \alpha \beta\left|z^{2}\right|}{1+|a|}-1>1+\lambda$. Consequently, $\left|z_{1}\right|>(1+\lambda)|z|$. In particular, $\left|z_{n}\right|>$ $|z|$. So we may apply the same argument repeatedly to find $\left|z_{n}\right|>(1+\lambda)^{n}|z|$. Thus, the orbit of $z$ tends to infinity. This completes the proof.

Corollary 4.6 Suppose that $|c|>\left(\frac{2(1+|a|)}{s \alpha}\right)^{\frac{1}{2}}$ and $|c|>\left(\frac{2(1+|a|)}{s \beta}\right)^{\frac{1}{2}}$, then the orbit of Jungck Ishikawa $\operatorname{IIO}\left(T_{c}, 0, \alpha, \beta, s\right)$ escapes to infinity.

In the proof of the theorem we used the facts that $|z| \geq|c|>\left(\frac{2(1+|a|)}{s \alpha}\right)^{\frac{1}{2}}$ and $|z| \geq|c|>$ $\left(\frac{2(1+|a|)}{s \beta}\right)^{\frac{1}{2}}$. Hence the following corollary is the refinement of the escape criterion discussed in the above theorem.

Corollary 4.7 (Escape criterion) Let $|z|>\max \left\{|c|,\left(\frac{2(1+|a|)}{s \alpha}\right)^{\frac{1}{2}},\left(\frac{2(1+|a|)}{s \beta}\right)^{\frac{1}{2}}\right\}$, then $\left|z_{n}\right|>(1+$ $\lambda)^{n}|z|$ and $\left|z_{n}\right| \longrightarrow \infty$ as $n \rightarrow \infty$. 
Corollary 4.8 Suppose that $\left|z_{k}\right|>\max \left\{|c|,\left(\frac{2(1+|a|)}{s \alpha}\right)^{\frac{1}{2}},\left(\frac{2(1+|a|)}{s \beta}\right)^{\frac{1}{2}}\right\}$ for some $k \geq 0$. Then $\left|z_{k+1}\right|>(1+\lambda)^{n}\left|z_{k}\right|$ and $\left|z_{k+1}\right| \longrightarrow \infty$ as $n \rightarrow \infty$.

This corollary gives us an algorithm for the generation of Julia sets and Mandelbrot sets of $T_{c} z=z^{3}+c$.

\subsection{Escape criterion for higher degree complex polynomials}

For higher degree complex polynomial $p(z)=z^{n}-a z+c$, we will choose $T z=z^{n}+c$ and $S z=a z$, where $n=2,3,4, \ldots, a$ and $c$ are complex numbers.

Theorem 4.9 Assume that $|z| \geq|c|>\left(\frac{2(1+|a|)}{s \alpha}\right)^{\frac{1}{n-1}}$ and $|z| \geq|c|>\left(\frac{2(1+|a|)}{s \beta}\right)^{\frac{1}{n-1}}$, where $0<$ $\alpha, \beta, s<1$ and $c$ is a complex parameter. Define

$$
\begin{aligned}
& S z_{1}=(1-\alpha)^{s} S z+\alpha^{s} T y, \\
& \vdots \\
& S z_{n}=(1-\alpha)^{s} S z_{n-1}+\alpha^{s} T y_{n-1},
\end{aligned}
$$

where

$$
\begin{aligned}
& S y=(1-\beta)^{s} S z+\beta^{s} T z, \\
& \vdots \\
& S y_{n-1}=(1-\beta)^{s} S z_{n-1}+\beta^{s} T z_{n-1},
\end{aligned}
$$

Sz is injective, $T z=z^{n}+c$ and $n=2,3,4, \ldots$, then $\left|z_{n}\right| \rightarrow \infty$ as $n \rightarrow \infty$.

Proof To prove the theorem, we follow the mathematical induction. For $n=2, T z=z^{2}+c$, so the escape criterion is $|z|>\max \left\{|c|, \frac{2(1+|a|)}{s \alpha}, \frac{2(1+|a|)}{s \beta}\right\}$. For $n=3, T z=z^{3}+c$, so the escape criterion is $|z|>\max \left\{|c|,\left(\frac{2(1+|a|)}{s \alpha}\right)^{1 / 2},\left(\frac{2(1+|a|)}{s \beta}\right)^{1 / 2}\right\}$. Hence the theorem is true for $n=2,3, \ldots$ Now suppose that the theorem is true for any $n$. Let $T z=z^{n+1}+c, z_{0}=z, y_{0}=y$ and $|z| \geq$ $|c|>\left(\frac{2(1+|a|)}{s \alpha}\right)^{\frac{1}{n}},|z| \geq|c|>\left(\frac{2(1+|a|)}{s \beta}\right)^{\frac{1}{n}}$ exist, then we have

$$
S y_{n-1}=(1-\beta)^{s} S z_{n-1}+\beta^{s} T z_{n-1}
$$

implies

$$
\begin{aligned}
|S y| & =\left|(1-\beta)^{s} S z+\beta^{s} T z\right| \\
& =\left|(1-\beta)^{s} a z+\beta^{s}\left(z^{n+1}+c\right)\right| \\
& =\left|(1-\beta)^{s} a z+(1-(1-\beta))^{s}\left(z^{n+1}+c\right)\right| .
\end{aligned}
$$

Using binomial series up to linear terms of $\alpha$ and $(1-\alpha)$, we get

$$
\begin{aligned}
|S y| & =\left|(1-s \beta) a z+(1-s(1-\beta))\left(z^{n+1}+c\right)\right| \\
& \geq(1-s(1-\beta))\left|z^{n+1}+c\right|-|(1-s \beta) a z|
\end{aligned}
$$




$$
\begin{aligned}
& \geq(s-s(1-\beta))\left|z^{n+1}+c\right|-|(1-s \beta) a z| \quad \text { because } s<1 \\
& \geq s \beta\left|z^{n+1}\right|-s \beta|c|-|a z|+|s \beta a z| \\
& \geq s \beta\left|z^{n+1}\right|-s \beta|z|-|a z|+|s \beta a z| \quad \text { because }|z| \geq|c| \\
& \geq s \beta\left|z^{n+1}\right|-s \beta|z|-|a z| \quad \text { because }|a| \geq 0
\end{aligned}
$$

gives us

$$
\begin{aligned}
|a y| & \geq s \beta\left|z^{n+1}\right|-|z|-|a z| \quad \text { because } s \beta<1 \\
& \geq s \beta\left|z^{n+1}\right|-|a||z|-|z| \\
& =s \beta\left|z^{n+1}\right|-(1+|a|)|z| \\
& =|z|\left(s \beta\left|z^{n}\right|-(1+|a|)\right) .
\end{aligned}
$$

Thus

$$
|y| \geq|z|\left(\frac{s \beta\left|z^{n}\right|}{1+|a|}-1\right) .
$$

Since $|z|>\left(\frac{2(1+|a|)}{s \beta}\right)^{\frac{1}{n}}$, it implies $|y|^{n+1}>|z|^{n+1}\left(\frac{s \beta|z|^{n}}{1+|a|}-1\right)^{n+1}>s \beta|z|^{n+1}$.

For $z_{0}=z$ and $y_{0}=y$, consider

$$
\left|S z_{n}\right|=\left|(1-\alpha)^{s} S z_{n-1}+\alpha^{s} T y_{n-1}\right|
$$

from which we obtain

$$
\left|S z_{1}\right|=\left|(1-\alpha)^{s} S z+\alpha^{s} T y\right|
$$

yields

$$
\begin{aligned}
\left|a z_{1}\right| & =\left|(1-\alpha)^{s} S z+\alpha^{s} T y\right| \\
& =\left|(1-\alpha)^{s} a z+(1-(1-\alpha))^{s}\left(y^{n+1}+c\right)\right| \\
& \geq(1-s(1-\alpha))\left|y^{n+1}+c\right|-|(1-s \alpha) a z| \\
& \geq s \alpha\left|y^{n+1}\right|-(1+|a|)|z| \\
& \geq s^{2} \alpha \beta\left|z^{n+1}\right|-(1+|a|)|z| \\
& \geq|z|\left(s^{2} \alpha \beta\left|z^{n}\right|-(1+|a|)\right) .
\end{aligned}
$$

Hence

$$
\left|z_{1}\right| \geq|z|\left(\frac{s^{2} \alpha \beta\left|z^{n}\right|}{1+|a|}-1\right)
$$

since $|z| \geq|c|>\left(\frac{2(1+|a|)}{s \alpha}\right)^{\frac{1}{n}}$ and $|z| \geq|c|>\left(\frac{2(1+|a|)}{s \beta}\right)^{\frac{1}{n}}$, so that $|z|>\left(\frac{2(1+|a|)}{s^{2} \alpha \beta}\right)^{\frac{1}{n}}$. Therefore there exists $\lambda>0$ such that $\frac{s^{2} \alpha \beta\left|z^{n}\right|}{1+|a|}-1>1+\lambda$. Consequently, $\left|z_{1}\right|>(1+\lambda)|z|$. In particular, $\left|z_{n}\right|>$ 
$|z|$. So we may apply the same argument repeatedly to find $\left|z_{n}\right|>(1+\lambda)^{n}|z|$. Thus, the orbit of $z$ tends to infinity. This completes the proof.

Corollary 4.10 Suppose that $|c|>\left(\frac{2(1+|a|)}{s \alpha}\right)^{\frac{1}{n-1}}$ and $|c|>\left(\frac{2(1+|a|)}{s \beta}\right)^{\frac{1}{n-1}}$, then the orbit $\operatorname{JIO}\left(T_{c}, 0\right.$, $\alpha, \beta, s)$ escapes to infinity.

Corollary 4.11 (Escape criterion) Suppose that $\left|z_{k}\right|>\max \left\{|c|,\left(\frac{2(1+|a|)}{s \alpha}\right)^{\frac{1}{n-1}},\left(\frac{2(1+|a|)}{s \beta}\right)^{\frac{1}{n-1}}\right\}$ for some $k \geq 0$. Then $\left|z_{k+1}\right|>(1+\lambda)^{n}\left|z_{k}\right|$ and $\left|z_{k+1}\right| \longrightarrow \infty$ as $n \rightarrow \infty$.

This corollary gives us an algorithm for the generation of Julia sets and Mandelbrot sets of $T_{c} z=z^{n}+c$.

\section{Conclusions}

In this paper, some fixed point results for Jungck Mann and Jungck Ishikawa iterations with $s$-convexity have been introduced in the study of Julia and Mandelbrot sets. The new escape criterions for complex quadratic, cubic and nth degree polynomials have been established. If we take $s=1$, it provides previous results existing in the relative literature.

Competing interests

The authors declare that they have no competing interests.

Authors' contributions

All authors contributed equally to the writing of this paper. All authors read and approved the final manuscript.

\section{Author details}

'Division of Science and Technology, University of Education, Lahore, Pakistan. ${ }^{2}$ Department of Mathematics and RINS, Gyeongsang National University, Jinju, 660-701, Korea. ${ }^{3}$ Department of Mathematics, Lahore Leads University, Lahore, 54810, Pakistan.

\section{Acknowledgements}

This research is supported by Gyeongsang National University, Korea.

Received: 26 May 2015 Accepted: 10 September 2015 Published online: 25 September 2015

\section{References}

1. Julia, G: Sur l'itération des functions rationnelles. J. Math. Pures Appl. 8, 737-747 (1918)

2. Mandelbrot, BB: The Fractal Geometry of Nature. Freeman, New York (1983)

3. Negi, A, Rani, M: A new approach to dynamic noise on superior Mandelbrot set. Chaos Solitons Fractals 36(4), 1089-1096 (2008)

4. Negi, A, Rani, M: Midgets of superior Mandelbrot set. Chaos Solitons Fractals 36(2), 237-245 (2008)

5. Rani, M, Kumar, V: Superior Mandelbrot set. J. Korea Soc. Math. Educ. Ser. D Res. Math. Educ. 8(4), $279-291$ (2004)

6. Rani, M, Kumar, M: Circular saw Mandelbrot sets. In: WSEAS Proc. 14th Int. Conf. on Applied Mathematics (Math '09): Recent, Advances in Applied Mathematics, pp. 131-136 (2009)

7. Devaney, RL: A First Course in Chaotic Dynamical Systems: Theory and Experiment. Addison-Wesley, Reading (1992)

8. Chauhan, YS, Rana, R, Negi, A: Complex dynamics of Ishikawa iterates for non integer values. Int. J. Comput. Appl. 9(2), 9-16 (2010)

9. Chauhan, YS, Rana, R, Negi, A: New Julia sets of Ishikawa iterates. Int. J. Comput. Appl. 7(13), 34-42 (2010)

10. Rani, M: Cubic superior Julia sets. In: Proc. European Computing Conference, pp. 80-84 (2011)

11. Rani, M: Superior antifractals. In: IEEE Proc. ICCAE 2010, vol. 1, pp. 798-802 (2010)

12. Rani, M: Superior tricorns and multicorns. In: WSEAS Proc. 9th Int. Conf. on Applications of Computer Engineering (ACE '10): Recent Advances \& Applications of Computer Engineering, pp. 58-61 (2010)

13. Rani, M, Agarwal, R: Effect of noise on Julia sets generated by logistic map. In: Proc. 2nd IEEE International Conference on Computer and Automation Engineering (ICCAE 2010), vol. 2, pp. 55-59 (2010)

14. Rani, M, Agarwal, R: Effect of stochastic noise on superior Julia sets. J. Math. Imaging Vis. 36, 63-68 (2010)

15. Rani, M, Agarwal, R: Generation of fractals from complex logistic map. Chaos Solitons Fractals 42(1), 447-452 (2009)

16. Rani, M, Kumar, V: Superior Julia set. J. Korea Soc. Math. Educ. Ser. D Res. Math. Educ. 8(4), 261-277 (2004)

17. Rani, M, Negi, A: New Julia sets for complex Carotid-Kundalini function. Chaos Solitons Fractals 36(2), $226-236$ (2008)

18. Kang, SM, Rafiq, A, Tanveer, M, Ali, F, Kwun, YC: Julia and Mandelbrot sets in Jungck Mann and Jungck Ishikawa orbits. Mitteilungen Klosterneuburg 65, 1 (2015)

19. Rana, R, Chauhan, YS, Negi, A: Non linear dynamics of Ishikawa iterates. Int. J. Comput. Appl. 7, 13 (2010)

20. Bernstein, F, Doetsch, G: Zur Theorie der konvexen Funktionen. Math. Ann. 76, 514-526 (1915) 
21. Breckner, WW, Orbán, G: Continuity Properties of Rationally s-Convex Mappings with Values in Ordered Topological Liner Space. Babes-Bolyai University, Kolozsvár (1978)

22. Hudzik, H, Maligranda, L: Some remarks on si-convex functions. Aequ. Math. 48, 100-111 (1994)

23. Alomari, M, Darus, M: On Co-ordinated s-convex functions. Int. Math. Forum 3(40), 1977-1989 (2008)

24. Alomari, M, Darus, M: Hadamard-type inequalities for s-convex functions. Int. Math. Forum 3(40), 1965-1970 (2008)

25. Kirmaci, US, et al.: Hadamard-type inequalities for s-convex functions. Appl. Math. Comput. 193, 26-35 (2007)

26. Dragomir, SS, Fitzpatrick, S: The Hadamard's inequality for s-convex functions in the second sense. Demonstr. Math. 32(4), 687-696 (1999)

27. Takahashi, W: A convexity in metric space and nonexpansive mappings, I. Kodai Math. Semin. Rep. 22, 142-149 (1970)

28. Ojha, DB, Mishra, MK: An application of fixed point theorem for s-convex function. Int. J. Eng. Sci. Technol. 2(8), $3371-3375(2010)$

29. Olatinwo, MO, Imoru, CO: Some convergence results for the Jungck-Mann and the Jungck-Ishikawa iteration processes in the class of generalized Zamfirescu operators. Acta Math. Univ. Comen. 77(2), 299-304 (2008)

\section{Submit your manuscript to a SpringerOpen ${ }^{\mathcal{O}}$ journal and benefit from:}

- Convenient online submission

Rigorous peer review

- Immediate publication on acceptance

- Open access: articles freely available online

- High visibility within the field

- Retaining the copyright to your article 